\section{DEL ANÁLISIS DEL TERRITORIO A LA POLÍTICA REGIONAL}

\section{Laura Victoria Arzayús Correa*}

Fecha de Recepción: 8 de Abril de 2010

Fecha de Aceptación: 30 de Abril de 2010

Artículo Resultado de Revisión

\section{Resumen}

Este texto está pensado como un ejercicio que identifica las bases conceptuales sobre las cuales deben erigirse las políticas regionales. Luego de la revisión de los componentes del territorio, se propone el proceso de análisis del enfoque ecosistémico como herramienta metodológica para un acercamiento a una política regional completa y funcional. En Colombia no existen políticas que faciliten la planificación del territorio, por lo que se propone que estas políticas partan de una base conceptual y metodológica similar para conformar una estructura primaria homogénea que facilite una aplicación funcional en términos legislativos y de gobernabilidad, incluyendo en ésta componentes sociojurídicos. Al finalizar se exponen algunas conclusiones y recomendaciones favorables con miras a establecer una visión prospectiva positiva de las necesidades del país en términos de desarrollo.

\section{Palabras Clave}

Política regional, Territorio, Ecosistema, Economía Sostenible, Urbano, Rural, Prospectiva.

Socióloga de la Universidad Nacional de Colombia, Maestrante en Planeación Urbana y Regional en la Pontificia Universidad Javeriana. Correo electrónico: larzayus@javeriana.edu.co; lauraarzayus@hotmail. com

\section{FROM THE TERRITORY ANALYSIS TO THE REGIONAL POLICIES}

\begin{abstract}
This text is thought as the development of an exercise that identifies the conceptual basis on which regional policies must be build. After the review of the territorial components, it is proposed the process analysis of the ecosystem approach as a methodological tool for a complete and functional regional policy.

In Colombia there are no policies in order to facilitate regional or territorial planning, therefore it is proposed that these policies begin from a similar conceptual and methodological base to form a homogeneous primary structure that, provide a functional application in legal and governability terms, including sociojuridic components. To conclude, it is expose some conclusions and favorable recommendations in order to establish a positive prospective view of the country's needs in terms of development.
\end{abstract}

\section{Keywords}

Regional policy, Territory, Ecosystem, Sustainable Economy, Urban, Rural, Prospective.

\section{INTRODUCCIÓN}

Actualmente nuestro país se encuentra en medio del desarrollo de políticas en el marco de un paradigma ${ }^{1}$ o enfoque interpretativo para el desarrollo de modelos regionales con características neo-institucionales. La lógica de organización social de gama amplia (característica del paradigma neo-institucional informa que las reglas de juego que guían

\footnotetext{
Cabe aclarar que algunos autores no comparten el calificativo de paradigma para aplicar al neoinstitucionalismo, considerando esta corriente como económica dentro de una economía que ya tiene sus propias bases de análisis; es decir, esta corriente no sería un paradigma en si, sino que compartiría los paradigmas de la economía basados en la racionalidad de los agentes económicos.
} 
el comportamiento de los agentes en una sociedad son fundamentales para explicar su desempeño económico) ${ }^{2}$ han generado en el país una corriente impulsadora de cambios y planes prospectivos para direccionar con objetivos claros el camino a seguir para un país en vía de desarrollo y con miras a involucrarse de manera protagónica en el mercado mundial de bienes y servicios.

Se podría pensar el desarrollo como:

"Un proceso con ciertas condiciones sociales previas, que atraviesa por etapas previsibles, requiere acumulación acelerada de capital e innovación tecnológica y empresarial, que conduce a la formación de sociedades y economías nacionales predominantemente urbanas e industriales, incluidas de actitudes modernizantes, capaces de elevar en forma permanente la producción de bienes $y$ servicios $y$, con el tiempo satisfacer ampliamente las demandas de consumo de sus miembros"3.

Según esto, es innegable que el primer paso para avanzar en el desarrollo debe ir dirigido hacia la creación de sistemas económicos estables, autosostenibles ambientalmente y funcionales, que articulen las dinámicas mixtas urbanas y regionales en los contextos de región, adaptando los resultados de los procesos históricos previos y proponiendo el desarrollo de visiones a largo plazo. Como dice Kalmanovitz, "creo que la aportación más importante que ha hecho el neoinstitucionalismo es a la historia económica, al plantear cómo las organizaciones sociales y los cambios históricos van construyendo una senda de

2 KALMANOVITZ, Salomón. El Neoinstitucionalismo como Escuela. PDF. Disponible online: http://www. banrep.gov.co/junta/publicaciones/salomon/ E1_\%20neoinstitucionalismo_como_escuela.pdf. Fecha de Consulta: 25 de Marzo de 2010

3 AGRUCO (Bolivia) - PRATEC (Perú). Agroecología y saber Andino. Taller Gráfico TAREA. Lima. 1990. p. 7. desarrollo económico que depende en gran medida del pasado"

El término de región ha sido ampliamente utilizado en diferentes contextos, y en el caso colombiano podemos pensar en regiones determinadas por características ambientales. Colombia tiene la ventaja estratégica de su localización geográfica, ya que su ubicación entre los trópicos "le permite recibir durante todo el año de manera es constante la irradiación solar, lo que, junto con la gran disponibilidad de agua, la notable productividad de los suelos y la vegetación y el gran mosaico de pisos térmicos y habitats que se encuentran en los tres ramales de la cadena montañosa de los Andes, ha permitido su colonización".

El gran mosaico de pisos térmicos que se menciona permite la caracterización y agrupación regional útil en términos de territorio según variables físicas de la siguiente forma ${ }^{6}$ :

1. Región caribe: con una extensión de 1.660 $\mathrm{km}$, desde la península de la Guajira hasta el Golfo de Urabá, incluyendo los territorios insulares de San Andrés y Providencia, esta zona presenta diversas características topográficas que incluyen desiertos, un macizo montañoso que asciende a $5.771 \mathrm{msnm}$, sabanas con ciénagas y lagunas y mar. El clima de esta zona es cálido en general, aunque en la cercanía al macizo las temperaturas pueden elevarse. Afortunadamente, a pesar de la colonización esta región presenta zonas naturales de conservación a nivel del suelo y en las zonas submarinas, además de un gran número de especies de flora y fauna.

4 KALMANOVITZ, Salomón. Ob. Cit. p. 1.

5 GÓMEZ, José Antonio. ORTEGA, Sergio Camilo. Biocomercio Sostenible - Biodiversidad y Desarrollo en Colombia. Instituto Alexander Von Humboldt. Bogotá. 2007. p. 23.

6 Caracterización basada en los detalles incluidos en el libro de GÓMEZ, José Antonio, et al. Ob. Cit. 
2. Región Pacífica: "El litoral del pacífico, donde la selva tropical se funde con un océano de aguas profundas, es una de las regiones menos pobladas de Colombia y por lo tanto, la naturaleza se encuentra casi virgen" $"$. Esta zona va desde la cordillera oriental de los Andes y la línea de costa del territorio sobre el océano pacífico, aproximadamente $1.300 \mathrm{~km}$ desde el norte del Departamento de Nariño hasta el Darién; la característica más puntual de este territorio es su pluviosidad, con registros de más de 9.000 $\mathrm{mm}$ anuales, lo que la convierte en una región húmeda por sus precipitaciones y su clima que oscila entre 24 y $28^{\circ} \mathrm{C}$. Por ser zona selvática sin mayor intervención antrópica presenta gran biodiversidad de flora y fauna, además de cultural y étnica por ser asentamiento de grupos afrodescendientes.

3. Región andina: Esta zona está delimitada por los tres ejes montañosos que se ramifican de la gran cadena andina que ingresa al territorio colombiano por el sur, además de incluir los valles de los ríos Cauca y Magdalena, configurando así sus límites dentro de la zona central del país. La accidentada topografía de esta región contribuye a la diversidad de pisos térmicos, lo que a su vez genera una amplia gama de usos del suelo para actividades agrícolas. De acuerdo a esto, en cada nivel de altura sobre el nivel del mar se presenta diversidad de flora y fauna, pero desafortunadamente esta zona es donde más se ha generado colonización y desarrollo de infraestructura urbana, causando así intervenciones antrópicas que han afectado de manera significativa las características intrínsecas del territorio.

4. Región Orinoquía: "abarca más de 230.000 km2, (el 20\% del territorio) y comprende la vasta zona localizada al nororiente del país que llega hasta el río Orinoco; es una llanura que se extiende hacia el oriente de los Andes con limitadas sabanas

GÓMEZ, José Antonio, et al. Ob. Cit. p. 29. de pastos naturales, salpicadas de matas de monte y bosques riparios y surcadas por caudalosos ríos que bajan de la cordillera" ${ }^{\text {. Casi no existen }}$ accidentes geográficos en esta región, por lo que su distribución territorial y temperatura es uniforme, con presencia de vegetales de pastizales y vegetación herbácea con árboles dispersos. La fauna y flora de esta región es similar a la del caribe y no presenta mayores asentamientos poblados.

5. Región amazónica: la selva amazónica es una llanura de mas de $6.800 .000 \mathrm{~km} 2,420.000$ correspondientes al territorio colombiano. Por ser zona selvática con poca intervención antrópica, los ecosistemas naturales presentan sus características naturales con uno de los mayores bancos genéticos de especies de flora y fauna del trópico.

También se han realizado ejercicios que determinan las regiones en términos económicos, sociales o políticos, pero es esencial pensar en el territorio físico no sólo en términos topográficos de caracterizaciones útiles de especializar, sino también en términos de la materialización de la existencia humana y de las intervenciones antrópicas que modifican el uso del suelo; "la amplitud de este significado exige mucha atención de nuestra parte, para no considerar al espacio como un fragmento: es una totalidad"9. Es interesante ver como en estos contextos de nuevas caracterizaciones de las regiones a través de los territorios determinados por relaciones de acciones sociales, de flujos económicos o de escenarios jurídicos, aparecen claramente establecidas relaciones de poder y gobernabilidad orientadas por intereses de consolidación regional bajo estructuras

Ibíd. p. 36.

9 MANCANO FERNANDES, Bernardo. "Territorio, teoría y política”. En: LOZANO VELÁSQUEZ, Fabio. FERRO, Juan Guillermo (Editores). Las configuraciones de los territorios rurales en el siglo XXI. Pontificia universidad Javeriana. Bogotá. 2009. p. 40. 
organizacionales, establecidas por conflictos que se desarrollan dentro y fuera de los límites de la legalidad. Estas relaciones, muchas veces sin querer, son la base de la conformación de una estructura, pero no contribuyen a su desarrollo ni abandonan los límites de especialidad física o conceptual dentro de los que son concebidos.

Ha sido evidente que estas tensiones sociales de poder que se desarrollan en los territorios son determinantes para su caracterización e influyen en sus procesos de desarrollo, pero muchas veces no son tenidos en cuenta para una organización urbana-regional que implique diferentes aspectos organizacionales legislativos y judiciales; únicamente desarticulando los contenidos de una región no se avanza hacia la implementación de políticas que orienten su desarrollo.

Tal como lo expone Mancano:

"En los "abordajes territoriales" predominan los análisis de la dimensión económica y de la social, junto con una acepción de territorio como unidad geográfica determinada, casi siempre como un espacio de gobernancia. La definición de "territorio" por parte de órganos gubernamentales y agencias multilaterales no consideran las conflictividades de los diferentes tipos de territorio contenidos en el "territorio" de un determinado proyecto de desarrollo territorial" 10 .

El sesgo en el pensamiento y concepción de los que se deriva la planeación del territorio que debe surgir de la organización regional, posterior a su definición, es evidente cuando a la hora de legislar no se obtienen resultados prácticos para promover el desarrollo de las regiones, sobre todo en términos económicos. Si para lograr un fin determinado (en este caso la planeación funcional), del cual se tiene en claro el punto de partida, se desvía del camino el proceso de construcción conceptual del cual

$10 \quad$ Ibíd. p. 41. derivamos las conclusiones para los planes de acción, caemos en el vicio de juzgar el resultado como no viable sin evaluar cuales fueron las variables del proceso que nos desviaron el rumbo; así, sin una estructura clara del proceso, seguiremos desaprovechando herramientas que nos permitirán articular territorios, regiones y zonas alrededor de políticas regionales que contribuyan, aceleren y garanticen procesos satisfactorios de desarrollos sostenibles que aprovechen los ecosistemas naturales y urbanos, y las interrelaciones que entre ellos hemos construido en el marco de la sostenibilidad ambiental.

\section{La Conformación Territorial: lo rural}

El rigor para los procesos que aquí se proponen debe partir de una base prospectiva, una visión, pensando en el desarrollo que queremos y deseamos, buscando su plausibilidad en los términos en los cuales se desea.

En ocasiones, para organizar el territorio sólo se parte de las premisas de la organización político-administrativa y jurídica del país, pero mas adelante veremos como la descentralización administrativa en los términos en los que está planteada conceptualmente no es obstáculo para proceder a pensar el territorio en otros términos más favorables. Las nociones de la división político administrativa se han vuelto determinantes en términos de soberanía y gobierno, y para analizar esta relación podemos pensar en el concepto de soberanía como la "supremacía atribuida a un poder, grupo u orden jurídico"; pero aquí, para esta competencia, ampliamos la definición hacía el términos específico de soberanía política, "que es el monopolio del Estado para disponer de una coacción incondicionada dentro de los límites de su competencia jurídica" ${ }^{11}$.

11 Ver definición de Soberanía en: PRATT, Henry. Diccionario de Sociología. Fondo de Cultura Económica. México. 1997. 
Si pensamos en las relaciones de poder y la dominación que de la mencionada clasificación del territorio nos justifica, vemos como de esta manera se ostenta la soberanía a través del poder que se manifiesta en la dominación del territorio; esta dominación es dominación legal, en donde su tipo más puro es la dominación burocrática, ya que "su idea básica es: que cualquier derecho puede crearse y modificarse por medio de un estatuto sancionado correctamente en cuanto a la forma... Se obedece, no a la persona en virtud de su derecho propio sino a la regla estatuida, la cual establece al propio tiempo a quien y en que medida se deba obedecer" 12 ; para nuestro caso, todo lo derivado desde y hacia las consecuencias de la democracia.

Aparte de encontrarnos con los aspectos menos positivos de la influencia de las relaciones de poder a la hora de organizar un territorio, teniendo en cuenta que es una tendencia de dominación utilizar la división político administrativa dictada por las normas legales, nos encontramos también con el obstáculo de pensar en regiones con características urbano-regionales.

Para nadie es un secreto que el poder legítimo se ejerce desde los núcleos urbanos, pero en nuestro país vivimos un conflicto que genera regiones de dominio de fuerzas armadas que buscan legitimidad en luchas políticas desde las zonas rurales. Este conflicto causa en cada zona donde está presente un nivel diferente de impacto, y si pensamos la organización regional en términos prospectivos de desarrollo a todos los niveles, debemos considerar que para el momento de nuestro escenario estas situaciones deben estar resueltas juridicamente, teniendo en cuenta su importancia para la determinación de los roles de las zonas rurales dentro del conjunto regional territorial.

12 WEBER, Max. Economía y Sociedad. Fondo de Cultura Económica. Bogotá. 1997. p. 707.
Mancano es conciso cuando afirma que:

"Es interesante observar que el concepto de territorio se utiliza como un instrumento de control social para subordinar comunidades rurales a los modelos de desarrollo de las transnacionales. En sus diferentes acepciones, el territorio siempre se estudió a partir de las relaciones de poder, desde el Estado, el capital o diferentes sujetos, instituciones y relaciones. La esencia del concepto está en sus principales atributos: totalidad, soberanía, multidimensionalidad y multiescalaridad (diversas escalas geográficas). Por tanto, es imposible comprenderlo sin concebir las relaciones de poder que determinan la soberanía" ${ }^{13}$.

Con el progresivo y permanente crecimiento de la población, aumenta la demanda en bienes y servicios, y así mismo se incrementa la presión sobre los recursos naturales y la biodiversidad, ocasionando a lo largo del tiempo y del espacio, cambios cuantitativos y cualitativos, muchos de ellos adversos. El campo se ha convertido, por sus características naturales no sólo de producción sino también de conservación, en elemento de continua discusión; para llegar en algún punto a la determinación regional, debe pensarse en las cualidades principales bajo las cuales se piensa, gobierna y legisla en estas zonas actualmente: economía y desarrollo sostenible, sin obviar que de estas se derivan muchas otras.

Para empezar, podemos acercarnos a un territorio rural pensado hacia las zonas de protección que lo conforman (si en este existen) si lo enmarcamos en términos de zona de desarrollo sostenible, porque aunque en nuestro país existen zonas cuya vocación del suelo es únicamente protección, por ellas se transita y en ellas se realizan actividades, y los asentamientos causan afectaciones antrópicas que modifican las características intrínsecas naturales de estos territorios. Si

13 MANCANO FERNANDÉS, Bernardo. Ob. Cit. p. 42. 
para este ejercicio pensamos que el desarrollo sostenible debe estar determinado por una política de gestión ambiental, entendida como un proceso técnico, administrativo, financiero y político por medio del cual las autoridades encargadas organizan un conjunto de recursos de diversa índole (humanos, financieros, técnicos, de información, etc.) que tienen como finalidad la protección, manejo y preservación del medio ambiente y de los recursos renovables en un territorio específico, aparece la sostenibilidad como el término que plantea la necesidad de hacer compatible el crecimiento de la producción agropecuaria con el mantenimiento de la capacidad productiva de los recursos naturales; dicha condición requiere el establecimiento de una planificación sustentada en el conocimiento de las características propias de los recursos, apoyada en la formulación de estrategias de producción sostenible.

Por otro lado, se debe tener en cuenta que las ciudades importan servicios ecológicos desde regiones distantes y dependen de las transformaciones ecológicas que ocurren a escala global. Los cambios en las condiciones ecológicas asociados a la urbanización afectan los servicios ecológicos locales y globales, como así también su habilidad para sostener a la población urbana. Los patrones de desarrollo urbano afectan la cantidad y el patrón de áreas construidas y áreas naturales, semi- naturales o agrícolas, así como el uso de los servicios ecológicos en ecosistemas urbanos, por lo que argumentan que los distintos patrones urbanos (formas urbanas, distribución del uso de la tierra y conectividad) tienen efectos diferenciales sobre la resiliencia. El desarrollo urbano fragmenta, aísla y degrada hábitats naturales y su biodiversidad, simplifica y homogeneiza la composición de especies, interrumpe sistemas hidrológicos y modifica los flujos de energía y ciclos biogeoquímicos.

"La biodiversidad puede interpretarse como resultado de los procesos de intervención humana en el territorio, derivada de un proceso de selección artificial que tiene su origen en los cambios de cantidad y calidad de hábitat disponible para las poblaciones microbianas $y$ de flora y fauna silvestre, $y$ determinados por las leyes de la fisica, la química, la biología y las decisiones politicas de los humanos" 14 .

Al hablar de biodiversidad, también es importante hablar de su relación con aquellos procesos físicos que no requieren participación de los seres vivos, lo que genera una conexión conocida como tejido o red territorial. La integración de la biodiversidad al ordenamiento territorial evidencia la necesidad de evaluar y desarrollar políticas de conservación dirigidas a preservar determinados espacios (zonas protegidas), así como especies y razas biológicas.

"La fragmentación de los hábitats silvestres y su degradación representan el continuo declive de lugares considerados reservorios de biodiversidad. Estos sitios son, hasta cierto punto, una garantia de permanencia de la 'naturaleza' y de las posibilidades de su mantenimiento y recuperación. La interconexión de estos ambientes es una tarea de interés global. Las politicas de conservación basadas en la consideración de especies biológicas o porciones del territorio aislados son inadecuadas a largo plazo. Los patrones de dispersión y migración deben ser salvaguardados reconociéndose sus conexiones territoriales. Este constituye el principal reto de la integración entre las infraestructuras de desarrollo y el tejido territorial. En otros casos, los hábitats pueden mantener mecanismos propios de autorregulación demográfica y su mantenimiento depende precisamente de su

14 BAPTISTE, Brigitte Luis Guillermo. RINCÓN, Sofía. "Planificación de la Biodiversidad en los procesos de configuración local del territorio". En: LOZANO VELÁSQUEZ, Fabio. FERRO, Juan Guillermo (Editores). Las configuraciones de los territorios rurales en el siglo XXI. Pontificia universidad Javeriana. Bogotá. 2009. 
aislamiento, de manera que la conexión constituye un inconveniente" 15 .

Es aquí donde se hace necesario involucrar el concepto de Enlaces como elementos ecológicos del paisaje y Conectividad del territorio. Los enlaces corresponden a sendas para el desplazamiento de animales y plantas a través de ambientes inhóspitos, y además juegan un papel ecológico más amplio debido a que prestan otros beneficios para la conservación como sustentar individuos residentes o poblaciones de animales y desempeñar un papel clave en mantener la diversidad de la vida silvestre y la continuidad de procesos ecológicos en ambientes muy modificados.

Por su parte, la conectividad territorial vista desde la perspectiva ecológica, podría definirse como la capacidad del paisaje de mantener los flujos y las conexiones entre los diferentes elementos, y adicionalmente como la capacidad que tiene una población de una determinada especie para desplazarse a través del paisaje, dados tanto el nicho ambiental de la especie en cuestión, como la distribución espacial de sus poblaciones, y la heterogeneidad espacial del territorio. La conectividad obedece a un atributo extrínseco del paisaje porque debe ser valorado para cada especie, y además contempla el elemento espacial porque detecta conexiones funcionales entre distintas localizaciones del paisaje.

Los espacios naturales protegidos pueden considerarse como los territorios sobre los que la sociedad cuenta con más fuerza para imponer un criterio de protección de los procesos ecológicos naturales frente a otros territorios donde prima la explotación y otras

15 DÍAZ, Francisco. SCHMITZ, María. "Tramas Espaciales del Paisaje. Conceptos, Aplicabilidad y Temas Urgentes para La Planificación Territorial". En: Conectividad Ambiental: Las Áreas Protegidas en la Cuenca Mediterránea. Consejería del Medio Ambiente, Junta de Andalucía - UICN. Andalucía. 2003. actividades. Sin embargo, la estructura actual de los espacios protegidos no asegura la necesaria conectividad funcional del conjunto del territorio, por lo que es necesario abordar un nuevo enfoque más integral. Dada la relevancia de la superficie actualmente protegida, así como la experiencia ganada en la última década en la planificación y gestión de estos territorios, cobra sentido el objetivo de iniciar el proceso de construcción de un sistema de conservación tomando estos espacios como punto de partida.

Los nuevos enfoques para la planificación y gestión de los espacios naturales deben contemplar el mantenimiento de los ecosistemas naturales y semi-naturales, dando prevalencia al objetivo de la conservación de los bienes y servicios ambientales que proveen a la sociedad, y no sólo a la conservación de especies o espacios singulares o representativos. Cada vez son más los países que han iniciado el proceso de redefinición de sus sistemas de conservación de la naturaleza. La aproximación de las redes ecológicas supone el paso a una estrategia activa decidida de integración de los objetivos de conservación de la naturaleza en sentido amplio en la planificación del conjunto del territorio. Todas las iniciativas se han desarrollado en respuesta a la intensificación del uso del territorio causada por presiones similares, tecnológicas y económicas.

La fragmentación del paisaje (debido fundamentalmente a la proliferación de grandes infraestructuras de diversa índole y a la intensificación de los usos del suelo) se identifica como una de las principales causas de la pérdida de integridad del paisaje, por lo que las redes o sistemas de conservación deben tender a minimizar sus efectos.

Aparece aquí la gestión ambiental y su correspondiente legislación jurídica, como un proceso orientado a obtener resultados que debe integrar la planeación, la ejecución y la 
evaluación continua, e igualmente articular estratégicamente las acciones del ente gestor a su misión y sus objetivos ${ }^{16}$. La legislación ambiental se ha vuelto entonces esencial para regular las actividades sociales en términos de intervención de la naturaleza, a fin de promover la conservación de los recursos, problema que atañe y se ha convertido en objeto de estudio de múltiples disciplinas que, con miras al futuro, se han percatado del protagonismo de los ecosistemas naturales en todos y cada uno de los procesos del hombre, pasando por fin la sociedad desde el estado anti-ambientalista de la época de industrialización al estado proteccionista que continúa encaminando sus acciones a través de la legislación propia del ejercicio del poder de dominio (gobierno), ya que la protección del ambiente está regido por leyes ambientales fundadas en el principio de que este es patrimonio común y debe ser protegido en la búsqueda del bien general sobre el bien particular.

Para entenderlo mejor, pensemos en que:

"La solución de los problemas ambientales se conjugan agregados complejos y múltiples de ciencias humanas y ciencias fácticas. Son pues necesidades sociales inminentes las que exigen un tratamiento interdisciplinario y prospectivo de la problemática derivada de la degradación ambiental. La Legislación Ambiental es la expresión de esta interdisciplinaridad normativa en el tratamiento del ambiente, en donde en todos y cada una de sus regulaciones se pone de relieve el singular papel del Derecho" 17 .

$16 \quad$ Et al. "Modulo 1: Conceptos básicos sobre medio ambiente y desarrollo sostenible". En: Serie de Cartillas para el manejo ambiental municipal con participación ciudadana. Programa de fortalecimiento municipal y de Ong`s para el manejo ambiental hacia el año 2000. Fundación foro nacional por Colombia. Cali. 2001.

17 ROJAS, Germán Eduardo. Política y Legislación del Medio Ambiente. Ediciones Futuro. Bogotá. 1979. p. xiii.
Así las cosas, en el marco de una política de desarrollo urbano regional, debe establecerse una normatividad ambiental articulada con las normas y leyes de zonificación de usos del suelo, en el que además se otorgue prevalencia a la preservación de las zonas con altos índices de biodiversidad, se armonicen los usos del componente forestal, se incentive la creación de enlaces ecológicos que permitan una conectividad de los seres vivos con su hábitat (dando prioridad por ejemplo a aquellas especies que se encuentran en peligro de extinción).
"Respecto del principio prospectivo de las normas ambientales, el Dr. Carrizosa Umaña expresó lo siguiente: "El ambiente y los recursos naturales se deben utilizar según criterios de equidad que aseguren: primero, el desarrollo armónico del hombre $y$ de dichos recursos; segundo, la dispo- nibilidad permanente de ellos, y tercero, máxima participación social. Lo que se busca entonces es no solamente la justicia a secas. Además se busca la equidad y el equilibrio entre el desarrollo del hombre y de los recursos, se persigue el hecho de que estos existan permanentemente y se exige la participación de todos los miembros de la comuni- dad. ¿Para qué? Para el bienestar de los presentes y futuros habitantes del territorio. Es nada mas ni nada menos que la búsqueda de la justicia bio- lógica entre los diferentes organismos, la exigencia de la justicia social y la creación de la justicia en el tiempo, o sea la distribución equitativa del bienestar entre los que vivimos hoy y los que han de vivir mañana"18.

Una vez abordado de manera general el territorio rural en términos legislativos hacia la protección de los recursos, pasemos a pensarlo en los términos de su importancia económica. La etapa de industrialización generó la maquinización de los procesos agrícolas, 1o que contribuyó al crecimiento de las ciudades por el desplazamiento de la mano de obra que se adhirió a las economías de escala de

$18 \quad$ Ibíd. p. 7. 
las empresas de las ciudades; esto causó un cambio en la significación de los procesos rurales en el marco de la economía regional.

Aquí pasamos nuevamente a retomar las implicaciones de los conceptos de multiescalaridad y multi-dimensionalidad en el territorio; cuando este

"...es concebido como uno solo, o sea como espacio de gobernancia, y se ignoran los otros que existen dentro del espacio de gobernancia, tenemos entonces una concepción "reduccionista", concepto que sirve más como instrumento de dominación por medio de las politicas neoliberales ${ }^{19}$. En esta condición, una determinada región es escogida para la implantación de politicas de desarrollo, en gran parte a partir de los intereses del capital. Las comunidades campesinas, evidentemente, tienen menor poder politico $y$, por tanto, tendrán menor poder de decisión en la determinación de las politicas, por más que el discurso de las instituciones defienda el "empoderamiento" de las comunidades rurales. De este modo, las politicas promueven el fortalecimiento de las relaciones capitalistas en detrimento de las no capitalistas, familiares y comunitarias. Así se intensifican las politicas de expropiación de comunidades rurales, que pierden sus territorios para el capital, que necesita apropiarse continuamente de los territorios campesinos para su expansión" 20 .

19 En este contexto aparece el término neoliberal referido a modelos socioeconómicos cuyo objetivo principal sería ser facilitadotes para que los grandes capitales internacionales operen con tranquilidad. A fin de que esta situación se genere, se buscará estabilizar y controlar las economías, principalmente mediante el control de la distribución de la riqueza. También se pretenderá buscar la estabilidad de los flujos de capitales a través de la especulación. Para profundizar se puede ver a: GIL RIVERO, José. La Dominancia del Paradigma Neoliberal. PDF. Disponible online: www. laberinto.uma.es. Fecha de consulta: 8 de Abril de 2010 .

20 BAPTISTE, Brigitte Luis Guillermo, et al. Ob. Cit. p. 447.
Los territorios rurales adquieren entonces la importancia estratégica como territorios en los cuales a nivel de relaciones de poder se surten conflictos de intereses y dominación, y entran a jugar el papel de "regiones económicas que se configuran de forma funcional en la concordancia de un centro y su zona de influencia"21; algunos podríamos decir que se convierten en centralidades protagonistas a nivel económico, alrededor de las cuales se generan flujos y dinámicas orientadas a controlar y participar activamente en las etapas de comercialización de los productos fruto de las actividades de producción.

Entonces, los criterios económicos para caracterizar las zonas rurales (y las urbanas también) pueden llegar a convertirse en una herramienta válida si se tiene en cuenta que el desarrollo sostenible de las actividades económicas de una determinada región es una meta plausible para la implementación de estrategias que contribuyan al desarrollo de políticas regionales. Pero, esta caracterización debe realizarse en principio a nivel conceptual en el marco de una política para posteriormente realizar la construcción de las variables particulares para la zona a la cual va dirigida, ya que en los casos en donde no están dinamizados los procesos económicos y el desarrollo de estos no se genere en un marco homogéneo y mientras existan zonas confluentes con diferentes niveles de atraso en estos aspectos de desarrollo, las políticas basadas en hechos concretos y no en procesos conceptuales previos se convertirán en herramientas de papel, no funcionales y terminarán siendo en muchos casos esfuerzos fallidos de legislación no apta para aplicar a nivel regional; es decir, a pesar de los esfuerzos seguiremos sin políticas articuladas en contextos territoriales a través de

21 EGNER, Erich. Política Regional y Desarrollo Económico. Ediciones Bilbao. Bilbao. Primer edición 1967. p. 118. 
las cuales se incrementen y apoyen los procesos de desarrollo económico.

Se debe tener en cuenta que existen tendencias para adoptar los territorios rurales en la función de su productividad: "una que se adapta a las limitaciones y tiempos de la naturaleza y otra que pretende sacar el mayor provecho por medio de la implementación de tecnologías intensivas $y$ efectivas a corto plazo"22. Hemos sido testigo que los procesos de industrialización capitalistas de la cultura occidental son los protagonistas de la segunda estrategia, pero actualmente en el marco de una actitud proteccionista del ambiente se están adelantando a nivel mundial estrategias y programas que proponen un acercamiento que unifique fines económicos con fines sostenibles, como es el caso del Biocomercio Sostenible, cuyos principios son:

"Conservación de la biodiversidad, uso de la biodiversidad, distribución justa y equitativa de los beneficios derivados del uso de la biodiversidad, Sostenibilidad económica, cumplimiento de la legislación nacional y acuerdos internacionales ratificados o adoptados, respecto a los derechos de los actores involucrados en el biocomercio y claridad sobre la tenencia de la tierra, el uso y acceso a los recursos naturales" 23 .

Esperemos que estas iniciativas perduren, se mantengan y se aumenten, generando así nuevas herramientas y campos de acción para la implementación de políticas de orden regional, que, como hemos visto hasta ahora, siguen encontrando campos de acción sin importar bajo que óptica o en qué contexto se ordenen los territorios. Es importante resaltar de nuevo, que para este ordenamiento se debe pensar en la ciudad-región como un todo que es más que la suma de sus partes.

Para pensar la región como un todo, se debe también tener en cuenta la necesidad de

22 GÓMEZ, José Antonio, et al. Ob. Cit. p. 51.

23 Ibíd. p. 67. articular las bases de una nueva institucionalidad sobre la ya existente, la cual permitirá tener en cuenta consideraciones sobre las fronteras, las cuales son cada vez menos definitivas entre las zonas rurales y urbanas; sobre los municipios, sobre los cuales no es necesario que recaiga únicamente el poder de dominación sobre la organización político administrativa; y sobre los procesos de descentralización, sus ventajas y desventajas para propender hacia una territorialización autónoma ${ }^{24}$, todo esto en el marco de los principios de el desarrollo sostenible y de una legislación soporte.

\section{La Conformación Territorial: lo urbano}

Es indiscutible que el hombre (que vive en los núcleos) ha alterado gran parte de los ecosistemas del planeta y ha contribuido a la pérdida de la biodiversidad. Sin ir más lejos: más de la mitad del agua dulce del planeta es utilizada por los seres humanos, aproximadamente el $50 \%$ de la superficie terrestre ha sido transformada por acciones antrópicas y la mayor parte del nitrógeno atmosférico es fijado por actividades humanas; podría decirse que, aunque el hombre no habite en todos los ecosistemas, de alguna forma los ha intervenido y alterado.

La urbanización ha transformado profundamente los paisajes, lo que inevitablemente ha producido efectos en la estructura, función y dinámica de los sistemas ecológicos en una amplia gama de escalas. Por ejemplo, las transformaciones de la tierra asociadas a la expansión urbana pueden afectar significativamente la biodiversidad, flujos energéticos, ciclos biogeoquímicos y condiciones climáticas a escala local y regional. Con una acelerada urbanización en el mundo se ha vuelto de suma importancia para los estudios ecológicos y

24 ECHEVERRI PERICO, Rafael, et al. Descentralización en el Agro. Ministerio de Agricultura y Desarrollo Rural en coedición con el Instituto Interamericano de Cooperación para la Agricultura. Bogotá. 1996. 
aplicaciones a gran escala (manejo de recursos naturales, planificación del uso de la tierra y conservación de la biodiversidad), considerar estas dramáticas transformaciones de la tierra y sus consecuencias ecológicas.

La urbanización es un fenómeno ecológico y social, y algunos de los estudios sobre ecología urbana promueven una investigación sobre la estructura y función de los ecosistemas en relación con el desarrollo urbano. Multiplicidad de estudios se desarrollan sobre el tema, sin embargo los más comunes se orientan a la comparación de diferentes tipos de uso del suelo en un establecimiento urbano, comparaciones de un área urbana con un área natural cercana, análisis de gradientes, estudios de las dinámicas de desarrollo urbano a través del tiempo y análisis de impactos ecológicos. Por otro lado, en algunos de los estudios ecológicos de ambientes urbanos se trata a las ciudades como otro bioma (conjunto de ecosistemas característicos de una zona biogeográfica que es nombrado a partir de la vegetación y de las especies animales que predominan en él y son las adecuadas ${ }^{25}$, donde son determinados únicamente con base en la presencia humana, característica determinante de estos territorios. Lo que la naturaleza es al componente rural de la región lo es el hombre al componente urbano de la misma.

Adicionalmente también se hace necesario incluir, para la caracterización de estos territorios, las descripciones cuantitativas de los sitios urbanos y sus atributos determinantes tales como la demografía, la geografía física, las tasa de cambio de los procesos ecológicos y el uso de la energía pueden ayudar a cuantificar y definir el ambiente urbano. Hasta hace

25 PICKETT, Steward. "Urban Ecological Systems: Linking Terrestrial Ecological, Physical, and Socioeconomic Components of Metropolitan Areas". En: MARZLUFF, John, et al. Urban Ecology: an International Perspective on the Interaction Between Humans and Nature. Springer Science. New York. 2008. pocos años las ciudades eran consideradas como ambientes artificiales, asumiendo que pocas especies pudieran sobrevivir en ellas y que las comunidades presentes eran fruto de la coincidencia. En la actualidad, la ciudad es vista como un ecosistema con patrones abióticos y bióticos definidos y con especies características que trascienden la escala local y regional; por esta condición particular de la ciudad se deben enfocar los análisis de las características de este componente de la región hacia la determinación de la abundancia y distribución de los organismos que componen los ecosistemas internos de estas zonas o ciudades enmarcadas en la definición de un territorio espacial.

Es indispensable que los procesos de planificación del territorio planteen estrategias para que el crecimiento urbano se articule en términos de región e incluya su componente rural para que no se generen más consecuencias ambientales que repercutan no solo en la sociedad sino en el medio en el que viven, y así mismo se evite que las externalidades negativas fruto de los procesos de intervención puedan trascender a escalas regionales y globales; de igual forma deben diseñar e implementar estrategias que mitiguen los impactos ya generados por causa de la expansión urbana.

La dinámica de crecimiento de los asentamientos urbanos en nuestro país ha sido fruto de dinámicas dispersas, lo que ha generado incrementos poblacionales desiguales en las diferentes regiones (si tomamos la caracterización realizada mediante los componentes topográficos del territorio); por ejemplo:

"El proceso de expansión urbana de la capital colombiana se caracterizó, en los últimos treinta años, por una progresiva saturación del espacio destinado a la construcción en el Distrito Capital, y un acelerado crecimiento poblacional de los municipios del norte y el occidente de la sabana, aledaños a Bogotá. Este fenómeno, identificado 
desde los años 90 y calificado de "metropolización" (DUREAU, FIÓREZ y HOYOS, 1993), obedece a una lógica gravitacional, caracterizada por un efecto de gradiente decreciente en la tasa de crecimiento, que es máxima en la periferia cercana a Bogotá, y disminuye con la distancia y el tiempo de acceso al $D C^{\prime 26}$.

Podemos decir que este es uno de los casos en donde el crecimiento poblacional de los centros urbanos se desarrolla hacia la periferia, generando zonas de transición entre los territorios urbanos y rurales, las cuales desarrollan funciones mixtas de acuerdo a su ubicación, y se convierten en fronteras indeterminadas. Ya el crecimiento y el desarrollo económico de nuestro país alcanzó un nivel que traspasó las concepciones territoriales establecidas y ahora genera la necesidad de articular ciudades-regiones o regiones, a fin de garantizar que las acciones de gobernabilidad se orienten hacia un desarrollo real y posible para una zona con las características naturales, sociales y económicas de un país en vías de desarrollo encaminado a insertarse en el capital mundial.

En este contexto aparece entonces el Capital Mundial Integrado, como una nueva forma conceptual para incluir en los sistemas sociales desde la economía mundial hasta la economía regional. Tal como lo argumenta Felix Guatari, los axiomas del CMI - cercamiento, des-territorialización de los antiguos espacios nacionales, regionales y profesionales, multicentralización, nuevas segmentaridades - jamás lograrán terminar con la evolución molecular de las ciudades ${ }^{27}$. A un espacio de re-territorialización como el que actualmente está en curso y que debe ser apoyado, le resulta

$26 \quad$ GOUËSET, Vicent. "Metropolización, poder local y cooperación territorial”. En: Et al. Hacer Metrópoli- La Región Urbana de Bogotá de Cara al Siglo XXI. Universidad Externado de Colombia. Bogotá. 2005. p. 67.

$27 \quad$ Ibíd. muy difícil no volverse funcional con respecto al deseo que lo ha constituido y no organizar la conexión de ese deseo al gran eje semiotizador del capitalismo.

Pero, el desarrollo no debe limitarse a las consecuencias puramente económicas que definen a un país en estos términos; debemos incluir el desarrollo tecnológico como parte esencial de este proceso y vehículo para su puesta en marcha; éste debe interrelacionarse con los conceptos de territorio:

\section{"Los espacios del capitalismo contemporáneo ya no se adhieren a los terruños, a las castas, a las tradiciones étnicas, religiosas, corporativas "pre- capitalistas" y cada vez menos a las metrópolis, a las ciudades industriales, a las relaciones de clase y a las burocracias del capitalismo segmentario de la era de los estado-nación. Estos espacios están confeccionados tanto a escala planetaria como a escala microsocial y microfisica" 28 .}

\section{La Descentralización}

Luego del avance a través de las posibles caracterizaciones de los componentes territoriales y las funciones que se interrelacionan en cada uno de los ecosistemas que existen en el concepto de región, veamos cómo esta construcción rigurosa es la base fundamental y esencial para la construcción de políticas de índole regional, y como estas adquieren gran importancia para el desarrollo de un país. Colombia aún se encuentra en una fase de desarrollo de un proceso de descentralización, cuyos componentes facilitan teóricamente el desarrollo de políticas de orden regional, con las características de influencia que anteriormente hemos descrito, entre muchas otras a las que debe ir esta orientada. Actualmente, podríamos decir que nuestro gobierno propende por lograr "un Estado moderno y eficiente,

28 GUATARI, Félix. Plan Sobre el Planeta. Traficantes de sueños. Madrid. 2004. p. 94. 
garante de todos los ciudadanos clientes, socializador del poder y de la acción económica, árbitro $y$ mediador en el conflicto, que garantice aquellos derechos de la población que no son negociables en el mercado, con cobertura y presencia en todos los rincones del país" 29 .

Para lograr estas metas se han propuesto caminos de diversa índole, y para el caso de la organización territorial en términos regionales con miras al desarrollo económico sostenible aparece la descentralización como una de las herramientas funcionales para llevar a cabo este proceso, cuyo marco institucional aparece en la Constitución de 1991 y funciona como "un esquema de descentralización política y administrativa, donde las entidades territoriales asumen un nuevo papel, un nuevo régimen de seguridad social, y un nuevo espacio para la acción privada, entre otros" ${ }^{30}$. Pero, este proceso ha generado tensiones por los cambios de planificación del modelo centralista anterior con su esquema sectorial, los cuales han generado crisis por no haber completado completamente el proceso de cambio.

Por causa de esta situación y por contar con lineamientos claros y concisos y con la culminación del proceso de descentralización es como

"...ahora conviven en Colombia el modelo de planificación sectorial centralizado con modelos integrales de planificación local y departamental. En el plan de desarrollo municipal la visión integral está por encima de la visión sectorial: por ejemplo, el desarrollo rural debe resolver

29 CASTAÑEDA ROCHA, María Consuelo. "Metodología del Balance social”. En: Et al. Presente y Futuro de la Politica Social. Cuarto Congreso Nacional Cooperativo. Confederación de Cooperativas de Colombia. Bogotá. 1996. p. 271.

30 CÁRDENAS GUTIÉRREZ, Jorge. "La política social y el Sector Agropecuario Colombiano". En: Et al. Presente y Futuro de la Política Social. Cuarto Congreso Nacional Cooperativo. Confederación de Cooperativas de Colombia. Bogotá. 1996. p. 95. problemas de salud, educación, vivienda, saneamiento básico, e inclusive, de producción; en el nivel nacional, cada uno de estos problemas es un tema sectorial. En esa dualidad de modelos de planificación se pone en evidencia una de las improntas del proceso de asignación de recursos, uno de los espacios fundamentales de trabajo hacia delante" ${ }^{31}$.

Como podemos deducir, el problema no es que no se diseñen propuestas adecuadas a las necesidades de un sistema en constante cambio con intereses de evolución en términos de desarrollo, a veces falla la ejecución de las estrategias creadas y es necesario ver que no es por problemas de su construcción conceptual o de la determinación de sus campos de acción, sino que en ocasiones no se aclaran los límites de su intervención ni se establecen metas de cumplimiento en términos espacio-temporales.

Otra característica del proceso de descentralización ha sido su afectación que en varios niveles ha permeado las dinámicas rurales y urbanas, influyendo en algunos procesos propios de estas áreas. Esta intervención eventualmente puede ser perjudicial, si se involucra la descentralización en el desarrollo de procesos de organización territorial-regional y no se analiza su influencia. Esta situación la expone Echeverri al hablar de las tensiones que se generan en los siguientes términos:

"Las politicas sectoriales y la descentralización en Colombia han registrado tendencias modernizantes que privilegian lo urbano sobre lo rural. La experiencia descentralista a nivel de sectores se ha concentrado en agua potable, salud y educación, con avances tenues e incluso nulos en los demás sectores. Más aún, los énfasis que realizan las entidades nacionales del Estado, en las competencias y recursos transferidos a favor de las entidades territoriales, privilegian la prestación de los servicios públicos urbanos (domiciliarios o

31 ECHEVERRI PERICO, Rafael, et al. Ob. Cit. p. 3. 
para poblaciones nucleadas) y tienden a relegar las actividades y poblaciones rurales, consideradas "premodernas". Los discursos plantean la extensión de los beneficios del proceso descentralizador a todo el municipio, pero en la práctica las acciones e inversiones se concentran en los núcleos urbanos y sólo de manera residual se invierte en las zonas rurales. En este enfoque se desconoce la "ruralidad" de la gran mayoría de los municipios del país y no se guarda la debida integralidad del proceso de desarrollo regional que exige un equilibrio urbano-rural. Esta situación se explica, quizás, por las dificultades institucionales para atender las poblaciones rurales, por definición dispersas y con necesidades especificas" 32 .

Seguramente esta explicación basada en distancias y dispersión resulta satisfactoria para quienes protagonizan los procesos, pero puede llegar a resultar insuficiente para quienes se acercan a una visión integral del proceso.

Entonces, si se hace una apropiación de una idea a modo de conclusión de este proceso generada en otro espacio, se puede decir que

“...la descentralización y la regionalización configuran, entonces, dos tendencias inevitables frente a las cuales el sector agropecuario y rural no es ni podrá ser ajeno. Con las especificidades y complejidades que lo caracterizan, el futuro de este sector se enmarcará en dichas tendencias y desde ahora se requiere una evaluación y una discusión sólida sobre los derroteros que se deben seguir si se quiere estar a tono con los nuevos tiempos"33.

\section{E1 Enfoque Ecosistémico}

Este enfoque como método de análisis ofrece un proceso riguroso para una construcción conceptual que tenga en cuenta los componentes claves necesarios para articular elementos

$\begin{array}{ll}32 & \text { Ibíd. p. } 28 . \\ 33 & \text { Ibíd. p. } 18 .\end{array}$ que serán claves en el futuro como la política regional en su concepción conceptual.

Como se dijo anteriormente, los cambios ocurridos en el paisaje durante las últimas décadas del siglo XX han originado la degradación de numerosos hábitats afectando negativamente a la biodiversidad a una escala global. Entre los principales procesos de cambio global destacan el cambio en los usos del suelo, el cambio climático, el aumento en las concentraciones de $\mathrm{CO} 2$ y cambios en el ciclo bioquímica del nitrógeno. De todos estos procesos, el cambio de uso del suelo se considera la causa principal de la pérdida y degradación de numerosos hábitats naturales, y por lo tanto de la biodiversidad. A escala global, las transformaciones más importantes en el paisaje han ocurrido como consecuencia de la utilización de los hábitats naturales para el aprovechamiento del hombre; además en áreas altamente intervenidas por la acción antrópica, también se producen importantes cambios en los usos del suelo como consecuencia de la modificación de las prácticas agropecuarias. Uno de los problemas más comunes observados, es la utilización de las tierras en aquello para lo cual no tiene vocación, o su utilización en actividades por encima de su capacidad productiva; ambas situaciones producen desequilibrios negativos que se manifiestan en un deterioro progresivo de los recursos naturales y en general de la biodiversidad de cada territorio.

Para realizar un análisis integrado del territorio con miras a establecer regiones que permitan el desarrollo de políticas regionales, es necesario aclarar algunas definiciones conceptuales en las que se basan los procesos de análisis. En primer lugar, territorio lo podemos definir como el campo de relaciones e interdependencias entre factores diversos ${ }^{34}$, y considerar este concepto como el punto de partida (ya

34 MOLTÓ, Enrique. HERNÁNDEZ, María. Desarrollo Local, Geografia y Análisis Territorial Integrado: Algunos 
sea urbano o rural, cuyas diferenciaciones se expusieron anteriormente); paisaje en este contexto entonces se debe entender entonces como los varios tipos de imagen de un territorio desde el punto de vista escénico, ya sea esta imagen percibida o pintada, en cuanto a su esencia; también está el territorio en un sentido geográfico, que resulta de un conjunto de elementos espaciales ligados por relaciones de interdependencia; es, entonces, una porción de la superficie de la tierra.

También los paisajes pueden ser definidos como una asociación característica de patrones estructurales, funcionales y temporales, que responden a unas circunstancias y condiciones específicas de tiempo y espacio en la interacción de los factores formadores ${ }^{35}$. Para integrar la relación entre el análisis del territorio con todo lo que implica, debemos considerar al hombre y su cultura como agentes dinamizadores y transformadores de los ecosistemas naturales ${ }^{36}$ en el contexto de una ciudad entendida en términos de formas (naturales, arquitectónicas, urbanas, humanas, productivas, sociales) consolidadas en el espacio con un ánimo corporativo puestas para un uso público, colectivo y social ${ }^{37}$, aproximándonos a ecosistema en términos de la articulación del sistema natural y el sistema sociocultural, en el cual los componentes están relacionados e interactúan ${ }^{38}$.

Habiendo aclarado estos conceptos podemos pasar a describir el camino para el análisis. Una

Ejemplos Aplicados. Departamento de Análisis Geográfico Regional. Universidad de Alicante. Alicante. 2002.

35 ETTER, Andrés. Introducción a la Ecología del Paisaje. Instituto Geográfico Agustín Codazzi. Bogotá. 1990.

36 ANDRADE, Ángela. Aplicación del Enfoque Ecosistémico en Latinoamérica. CEM-UICN. Bogotá. 2007.

37 ÁlVAreZ, Carlos. Cartilla del Espacio Público No. 2. Departamento Administrativo Defensoría del Espacio Público, Alcaldía Mayor. Bogotá. 2006

38 ANDRADE, Ángela. Ob. Cit. teoría básica para revisar, analizar, estudiar y comprender el territorio y todo lo que comprende es el llamado enfoque ecosistémico, el cual consiste en una aproximación al manejo adecuado de los recursos biofísicos por parte de una sociedad, es decir, trata de acercarse a la relación hombre-ecosistema y la relevancia actual que contiene como método estructurado que determina los problemas presentes en esta interrelación y proponen caminos para la solución satisfactoria de estos de acuerdo a las necesidades de ambas partes ${ }^{39}$.

El enfoque ecosistémico es una herramienta metodológica para examinar determinada estructura natural y su función, relacionada directamente con la acción del hombre; para que este enfoque sea funcional, es necesario que en su desarrollo, aplicado a cada caso particular, se tengan en cuenta componentes teóricos y de análisis de información recolectada relacionados con el dominio de la ciencia de diversas disciplinas. Este factor es esencial, ya que es la recolección de los datos adecuados y su análisis correctamente elaborado la clave para la funcionalidad de este sistema.

Si la relevancia de la interdisciplinariedad y el papel de cada uno no se define desde la concepción de este análisis, su curso no seguirá el camino adecuado y es probable que los resultados sobre los cuales se basan algunas decisiones y políticas de ordenamiento no sean lo suficientemente veraces contrastadas con la realidad.

Habiendo aclarado esta condición necesaria para que el enfoque ecosistémico sea adecuado, debemos comprender ahora que la aplicación de la metodología en si no es determinante para un resultado óptimo; debe tenerse en cuenta que los resultados obtenidos están directamente asociados a dinámicas de relaciones entre cada componente y entre

$39 \quad$ Ibíd. 
cada grupo de componentes en un modelo que represente los niveles de integración.

Además, a la hora de recolectar información referente a interrelaciones que ocurren dentro de un determinado ecosistema o grupo de ecosistemas que en un sistema abierto complejo se comportan de diferentes maneras en un territorio determinado para organizarlo en términos de región intervenible por políticas derivadas de las formas de dominación y gobernabilidad y las relaciones de poder, se debe prever que los comportamientos cambian, fluyen y no son constantes. Identificar cada componente, su papel y las relaciones en las que interviene es un trabajo extenso pero necesario; la relación ecológica entre los factores formadores o atributos del paisaje y las interacciones que allí se dan es lo que llamamos propiedades emergentes del paisaje, que en un contexto topográfico son las que soportan los desarrollos del territorio ${ }^{40}$.

Entonces, ya a esta altura tenemos claras las consideraciones iniciales para el proceso de un análisis integrado: primero, claridad de conceptos; segundo, interdisciplinariedad; y tercero, flujos constantes entre los sistemas. Ahora, se impone tener en cuenta el componente humano; un estudio que refleje la interacción del hombre con su entorno debe primero comprender al hombre en el contexto de la sociedad en la cual se desenvuelve. Aquí aparecen factores clave determinantes para esta relación, dentro de los que se incluyen las motivaciones y necesidades por las cuales los seres humanos ocupan un territorio, interfieren en un ecosistema, en un paisaje.

La situación política, social, económica y ambiental (por nombrar categorías generales) de un grupo humano asentado en una zona en particular determina sus actividades; los valores históricos, ancestrales y culturales sobre

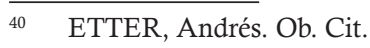

los cuales una sociedad ha basado su desarro1lo influye directamente en su relación con el entorno ${ }^{41}$. Históricamente los seres humanos han tenido la tendencia de agruparse, asentarse en un territorio y organizarse con fines productivos; esta condición humana ha generado un comportamiento sobre el medio ambiente en donde se ha hecho un aprovechamiento de los recursos naturales para subsistencia y actividades productivas, sin tener en cuenta la posibilidad de renovación de estos recursos y simplemente usarlos porque están allí. Actualmente a nivel mundial se vienen desarrollando gestiones orientadas a promover la preservación y determinar los usos y vocaciones del suelo, buscando frenar el desgaste desmedido de los recursos. En este aspecto el avance ha sido importante porque para las dinámicas del entendimiento del uso de la tierra se han tenido en cuenta la importancia de la dimensión humana y las fuerzas que a nivel global y regional y a nivel local están incluidas, determinando también el nivel de responsabilidad a la hora de tomar decisiones ${ }^{42}$.

Esta preocupación por las relaciones entre el hombre y el medio ambiente en un ecosistema, ha sido impulsada también por el crecimiento desmedido de la población mundial, la cual no se ha organizado sino que se ha apropiado de las ciudades en condiciones muchas veces no óptimas, que mezcladas con el poder de la economía global determinada por valores económicos y no por productos, ha desencadenado inconvenientes de salud pública y de seguridad alimentaria (entre otros). Esta acción del hombre de ocupar el territorio sin medir consecuencias ambientales debe ser tenida en

${ }_{41}$ MOLTÓ, Enrique, et al. Ob. Cit.

42 GEIST, Helmut. LAMBIN, Eric. What drives Tropical Deforestation. a meta-analysis of proximate and underlying causes of deforestation based on subnational case study evidence. LUCC Report Series No 4. LUCC - IHDP - Global Igbp Change. Belgium. Louvain-la-neuve, 2001. 
cuenta con miras hacia el futuro para buscar la forma de prevenir comportamiento nocivos similares, pero también el hombre debe preocuparse por el cómo cambia la ocupación de un territorio por acciones de la naturaleza; como elemento que define su conducta y se apropia de sus comportamientos, es el hombre quien debe definir las relaciones en el ecosistema sin sacar ventaja, y buscando establecer condiciones equitativas para los elementos que interactúan; es por esto necesario, para hallar el equilibrio, estudiar, pensar y repensar las relaciones entre hombre y medio ambiente.

Además, en el grupo de estas consideraciones debemos permitirnos el agregar una visión prospectiva que, basada en datos históricos, permita determinar hacia dónde va encaminada una sociedad, cuáles son sus necesidades, y como éstas promueven comportamientos y acciones hacia la intervención de un territorio.

Habiendo determinado el primer tramo de la ruta conceptual inicial que defina los pasos de un análisis, pasaremos a exponer la óptica sobre la cual se enmarcan los factores resultantes de la confluencia de los componentes de lo que debe ser un análisis integrado del territorio. Para este ejercicio es aplicable la teoría de los sistemas, la cual propone estudiar y establecer los patrones comunes de organización (o estructura de las relaciones) de los elementos componentes de una realidad ${ }^{43}$. La construcción de un modelo de esta teoría permite abstraer de la realidad cada una de las unidades ordenadas en una estructura sistémica de jerarquías, por las cuales se determinan los niveles de integración de un modelo.

Este modelo funciona en estos casos porque al estructurar un nivel de jerarquía surgen de la identificación de sus categorías las interrelaciones entre sus componentes que determinan la posición de cada objeto de estudio en el

$43 \quad$ ETTER, Andrés. Ob. Cit. nivel que le corresponde, caracterizando así el sistema de relaciones y sus condiciones.

La aplicación de esta herramienta en cada sistema, que se relaciona en un espectro abierto entre sistemas, facilita la obtención de información interna y externa de cada uno, clasificando las relaciones también dentro de patrones con componentes fenosistémicos y criptosistémicos para proceder a un análisis que permita un acercamiento conciso y explicativo de la relación de flujos, la conectividad y los factores determinantes de la relación estructura del paisaje-hombre con componentes naturales y antrópicos que han sido decisivos en los procesos de conformación del paisaje y ocupación de territorio por las sociedades, el cual se puede orientar y planificar hacia políticas de ordenamiento que reflejen las necesidades de cada uno de los integrantes de esa relación y los caminos para satisfacerlas en pro del beneficio común de todos y cada uno de los componentes del ecosistema, sin que ninguno se vea afectado en su función dentro de la estructura ni se vea perjudicado en su papel, ni se vea disminuida su capacidad de aportar, según sus características, al conjunto de un ecosistema autosostenible, conservable y funcional, que satisfaga las necesidades propias de las actividades naturales y humanas que en ese espacio se desarrollan.

Una vez aclarados los conceptos, pensada la estructura y determinados los campos y metodologías para la recolección de información, sigue el análisis de ésta, el cual se orientará a la búsqueda de las causas por las cuales surgen las relaciones de flujos y conectividad, las dinámicas internas y externas de los componentes de cada sistema y de cada uno de los sistemas entre sí, sin dejar de lado las consideraciones sociales que determinan el comportamiento humano; en este contexto, estas consideraciones permiten obtener imágenes de las dinámicas claras y concretas 
de la modificación de un territorio, de allí la importancia de su elaboración.

Cuando se han determinado estas dinámicas, se logra hallar el eje determinante y clave a través del cual se estructura en un solo sentido la información, orientándola a conclusiones aclaratorias y propositivas en cuanto a la transformación del paisaje se refiere, combinando tendencias de comportamiento hacia la obtención de visiones planificadoras funcionales aplicables a casos específicos en donde aparezcan datos similares a los recolectados para el análisis.

La tendencia mundial actual es clara en cuanto a la orientación de la finalidad de los estudios que impliquen enfoques ecosistémicos, ya que la noción de desarrollo está presente en todos los ámbitos; dentro de esta propuesta se debe pensar en términos de desarrollo sostenible como fruto de una equilibrada relación entre medio ambiente, sociedad y economía. Si se permite proponer ideas de futuros escenarios en donde estas condiciones estén predeterminadas y se consideren esenciales, se podrá pensar en un sistema sostenible cuanto sea capaz de utilizar la base existente sobre la que se sustenta el desarrollo, pero manteniendo la diversidad y reforzando los recursos ambientales ${ }^{44}$.

\section{CONCLUSIONES}

En primer lugar, debemos tener en cuenta que es casi una certeza que toda sociedad para poder ser viable necesita mínimo desarrollar satisfactoriamente seis campos. Primero, formación; necesita desarrollar conocimientos que, orientados a los servicios básicos, se

44 GUILlAMÓN, David. HOYOS, David. Movilidad Sostenible: de la Teoría a la Práctica. PDF. Disponible online: http://revistas.ucm.es/ghi/02119803/articulos/AGUC8989110271A.PDF. Fecha de consulta: 11 de Marzo de 2010. desarrollen a través de programas de investigación óptimos. Segundo y tercero, educación y salud a niveles accesibles por la totalidad de sus habitantes de acuerdo a sus necesidades. Cuarto, seguridad alimentaria garantizada. Quinto y sexto, un sistema productivo manejable y un nivel de gobernabilidad del sistema político y social que garantice la cobertura en términos equitativos ${ }^{45}$. Se impone un desarrollo estructural de un sistema social en el que se adhieran todos sus componentes, articulado a través de la interrelación entre regiones determinadas por territorios con características mixtas que se complementen con miras a ocupar cada uno de los roles necesarios para el desarrollo de una economía sostenible.

En segundo lugar, se debe pensar que para el desarrollo de la economía de una ciudad, es necesario el desarrollo de sus actividades agrícolas rurales en una simbiosis que favorezca a ambas partes; el comercio de productos de este tipo entre ciudades y entre regiones (regiones de un mismo país hasta regiones a nivel mundial, pensando la idea de abandonar las divisiones político-administrativas de fronteras como requisito necesario e indispensable en este desarrollo) garantiza los suministros necesarios de cada uno de sus productos; en los productos de consumo local y exportación de la seguridad alimentaria debe incluirse el conocimiento y la tecnología por el carácter protagonista que se propone imprimir en este proyecto, ya que sin los avances teóricos al mismo ritmo que los prácticos no es viable la conformación de una potencia en ese aspecto.

De esta manera, la ciudad crece por su relación con el campo y su propio proceso comercial, pero también el campo crece simultáneamente

WASSERMAN, Moisés, et al. Reflexiones Sobre la Ciencia y la tecnología: Colombia al iniciar el S. XXI. Simposio sobre ciencia, tecnología y desarrollo 1999. Academia Colombiana de Ciencias Exactas, Físicas y Naturales, Comisión Permanente para el Fomento de la Investigación Científica. Bogotá. 2001. 
por la necesidad de continuar produciendo para la satisfacción de las necesidades locales, y para la satisfacción de las demandas de exportación a otras regiones; "cuando más amplia es la economía local de una ciudad, mayor numero de productos inmediata o potencialmente exportables contiene" 46 .

En tercer lugar, es determinante para este desarrollo que se continúe desde la teoría acentuando la definición de conceptos claros que avancen en el cambio de mentalidad de los actores sociales. "Otra sociedad esta gestándose desde hoy mismo en los modos de sensibilidad, en los modos relacionales, en los vínculos con el trabajo, con la ciudad, con el medio ambiente, con la cultura, en una palabra, en el inconsciente social" ${ }^{\prime 4}$.

Además, tenemos otro tipo de necesidades que se deben incluir en este cambio. La política de ciencia y tecnología debe estar de acuerdo a la política de desarrollo económico de un país, deben ser elementos articulados estructurantes que soporten los proyectos actuales y futuros; la política de ciencia y tecnología acompaña al plan de desarrollo con énfasis en apoyar al sector productivo, particularmente a su capacidad exportadora ${ }^{48}$. "El desarrollo científico se convierte en el proceso gradual mediante el que esos conceptos han sido añadidos, solos y en combinación, al caudal creciente de la técnica y de los conocimientos científicos, y la historia de la ciencia se convierte en una disciplina que relata y registra esos incrementos sucesivos y los obstáculos que han inhibido su acumulación" ${ }^{49}$.

Pero, nada de esto funciona sin las políticas regionales correctamente orientadas por un proceso producto del rigor académico

46 JACOBS, Jane. La Economía de las Ciudades. Editorial Península. Barcelona. 1971. p. 123.

47 GUATARI, Félix. Ob. Cit.

48 WASSERMAN, Moisés, et al. Ob. Cit.

49 KUHN, Thomas. La Estructura de las Revoluciones Científicas. Fondo de Cultura Económica. Bogotá. 1998. y científico, alimentado con características determinantes conceptualmente que promueven un desarrollo de los estamentos oficiales a todos los niveles. Estos procesos son responsabilidad de quienes a través del ejercicio de la democracia ostentan el poder derivado de la legitimidad del Estado; debemos partir de la premisa de que los procesos de los gobiernos están fundamentados en los principios del bien común, teniendo en cuenta que "la acción social depende de un sistema de valores dado; así partimos de la premisa de que todas las acciones del hombre son guiadas por valores" 50 . A1 final no es en la metodología o en los resultados verificables de variables medibles con indicadores comprobables, es en el sistema de valores en el que debemos creer.

\section{BIBLIOGRAFÍA}

AGRUCO (Bolivia) - PRATEC (Perú). Agroecología y saber Andino. Taller Gráfico TAREA. Lima. 1990.

ÁlvarEZ, Carlos. Cartilla del Espacio Público No. 2. Departamento Administrativo Defensoría del Espacio Público. Bogotá. Alcaldía Mayor. 2006.

ANDRADE, Ángela. Aplicación del Enfoque Ecosistémico en Latinoamérica. CEM-UICN. Bogotá. 2007.

BAPTISTE, Brigitte Luis Guillermo. RINCÓN, Sofía. "Planificación de la Biodiversidad en los procesos de configuración local del territorio". En: LOZANO VELÁSQUEZ, Fabio. FERRO, Juan Guillermo (Editores). Las configuraciones de los territorios rurales en el siglo XXI. Pontificia universidad Javeriana. Bogotá. 2009.

CÁRDENAS GUTIÉRREZ, Jorge. "La política social y el Sector Agropecuario

50 CASTAÑEDA ROCHA, Maria Consuelo. Ob. Cit. p. 277. 
Colombiano". En: Et al. Presente y Futuro de la Política Social. Cuarto Congreso Nacional Cooperativo. Confederación de Cooperativas de Colombia. Bogotá. 1996.

CASAS, Andrés (Editor). Bases Biocomportamentales de la Política. Pontificia Universidad Javeriana. Bogotá. 2009.

CASTAÑEDA ROCHA, María Consuelo. "Metodología del Balance social". En: Et al. Presente y Futuro de la Politica Social. Cuarto Congreso Nacional Cooperativo. Confederación de Cooperativas de Colombia. Bogotá. 1996.

DÍAZ, Francisco. SCHMITZ, María. "Tramas Espaciales del Paisaje. Conceptos, Aplicabilidad y Temas Urgentes para La Planificación Territorial". En: Conectividad Ambiental: Las Áreas Protegidas en la Cuenca Mediterránea. Consejería del Medio Ambiente, Junta de Andalucía - UICN. Andalucía. 2003.

ECHEVERRI PERICO, Rafael, et al. Descentralización en el Agro. Ministerio de Agricultura y Desarrollo Rural en coedición con el Instituto Interamericano de Cooperación para la Agricultura. Bogotá. 1996.

EGNER, Erich. Política Regional y Desarrollo Económico. Ediciones Bilbao. Bilbao. Primer edición 1967.

Et al. "Modulo 1: Conceptos básicos sobre medio ambiente y desarrollo sostenible". En: Serie de Cartillas para el manejo ambiental municipal con participación ciudadana. Programa de fortalecimiento municipal y de Ong`s para el manejo ambiental hacia el año 2000. Fundación foro nacional por Colombia. Cali. 2001.

ETTER, Andrés. Introducción a la Ecología del Paisaje. Instituto Geográfico Agustín Codazzi. Bogotá. 1990.

GEIST, Helmut. LAMBIN, Eric. What drives Tropical Deforestation. a meta-analysis of proximate and underlying causes of deforestation based on subnational case study evidence. LUCC Report Series No 4. LUCC - IHDP - Global Igbp Change. Belgium. Louvain-la-neuve. 2001.

GIL RIVERO, José. La Dominancia del Paradigma Neoliberal. PDF. Disponible online: www.laberinto.uma.es. Fecha de consulta: 8 de Abril de 2010.

GÓMEZ, José Antonio. ORTEGA, Sergio Camilo. Biocomercio Sostenible - Biodiversidad y Desarrollo en Colombia. Instituto Alexander Von Humboldt. Bogotá. 2007.

GOUËSET, Vicent. "Metropolización, poder local y cooperación territorial". En: Et al. Hacer Metrópoli - La Región Urbana de Bogotá de Cara al Siglo XXI. Universidad Externado de Colombia. Bogotá. 2005.

GUATARI, Félix. Plan Sobre el Planeta. Traficantes de sueños. Madrid. 2004.

GUILlAMÓN, David. HOYOS, David. Movilidad Sostenible: de la Teoría a la Práctica. PDF. Disponible online: http:// revistas.ucm.es/ghi/02119803/articulos/ AGUC8989110271A.PDF. Fecha de consulta: 11 de Marzo de 2010.

JACOBS, Jane. La Economía de las Ciudades. Editorial Península. Barcelona. 1971.

KALMANOVITZ, Salomón. El Neoinstitucionalismo como Escuela. PDF. Disponible online: http://www.banrep.gov.co/junta/ publicaciones/salomon/E1_\%20neoinstitucionalismo_como_escuela.pdf. Fecha de Consulta: 25 de Marzo de 2010.

KUHN, Thomas. La Estructura de las Revoluciones Científicas. Fondo de Cultura Económica. Bogotá. 1998.

KUKLINSKI, Antoni. Aspectos Sociales de la Política y de la Planeación Regional. Fondo de Cultura Económica. México. 1981. 
MANCANO FERNANDES, Bernardo. "Territorio, teoría y política". En: LOZANO VELÁSQUEZ, Fabio. FERRO, Juan Gui1lermo (Editores). Las configuraciones de los territorios rurales en el siglo XXI. Pontificia universidad Javeriana. Bogotá. 2009.

MOLTÓ, Enrique. HERNÁNDEZ, María. Desarrollo Local, Geografía y Análisis Territorial Integrado: Algunos Ejemplos Aplicados. Departamento de Análisis Geográfico Regional. Universidad de Alicante. Alicante. 2002.

PICKETT, Steward. "Urban Ecological Systems: Linking Terrestrial Ecological, Physical, and Socioeconomic Components of Metropolitan Areas". En: MARZLUFF, John, et al. Urban Ecology: an International Perspective on the Interaction Between Humans and Nature. Springer Science. New York. 2008.

PRATT, Henry. Diccionario de Sociología. Fondo de Cultura Económica. México. 1997.
REVÉIS, Edgar. Poder e Información: El Proceso decisorio en Tres Casos de Politica Regional y Urbana en Colombia. Universidad de los Andes. Bogotá. 1977.

ROJAS, Germán Eduardo. Política y Legislación del Medio Ambiente. Ediciones Futuro. Bogotá. 1979.

Senado de la República. Documentos de Politica Pública - Piensa Colombia: Los Aportes de la Academia, Tomo 1. Universidad Nacional de Colombia. Manizales. 2008.

WASSERMAN, Moisés, et al. Reflexiones Sobre la Ciencia y la tecnología: Colombia al iniciar el $S$. XXI. Simposio sobre ciencia, tecnología y desarroIlo 1999. Academia Colombiana de Ciencias Exactas, Físicas y Naturales, Comisión Permanente para el Fomento de la Investigación Científica. Bogotá. 2001.

WEBER, Max. Economía y Sociedad. Fondo de Cultura Económica. Bogotá. 1997. 
